\title{
SIMILARITIES: GRB 940217, GBR 090926A AND GRB 980923
}

\author{
J.R. Sacahui ${ }^{1}$, M.M. González ${ }^{1}$, N. Fraija ${ }^{1}$, J.L. Ramirez ${ }^{1}$ and W.H. Lee $^{1}$
}

\begin{abstract}
Few GRBs clearly present distinct High-Energy (HE) component that evolve independent of the usual Band function that describes the prompt keV-emission. This component can be long or short lasting compared to the burst duration. However, most of models only explain the long duration HE component. We have developed a leptonic model on the external shock framework to describe the HE components. We propose that the short $\mathrm{HE}$ component that falls in the $\mathrm{MeV}$ regime in coincidence with the prompt phase and, the second long lasting GeVemission could be understood as synchrotron self-Compton (SSC) emission from the reverse and forward shock respectively. This model requires a magnetized jet and a reverse shock in the thick shell case. This model already has been proven in GRB 980923 and GRB 090926A, the former with a short duration $\mathrm{MeV}$-component and the latter with both long $\mathrm{GeV}$ - and short MeV- HE components. All fluxes, energies and durations are consistent with the observed values. Here, we apply this model to GRB 940217 that presented similarities with GRB 090926A and GRB 980923.
\end{abstract}

\section{Introduction}

Photons with energies $\geq 100 \mathrm{MeV}$ have been detected in some long and short gamma-ray bursts (GRBs). In some bursts, these photons arrived within the prompt emission as in GRB 941017 (Hurley et al. 1994; Winkler et al. 1995), but they can also be delayed as in GRB 090926A (Ackerman et al. 2011) or last even much shorter than the prompt emission as in GRB 980923 (González et al. 2012).

General hadronic (Asano et al. 2009; Dermer \& Razzaque 2010) and leptonic (Papathanassiou \& Meszaros 1996; Sari et al. 1996) interpretations have been discussed to explain these photons. Within the hadronic models, the two most investigated scenarios are synchrotron radiation from high-energy (HE) protons accelerated in the relativistic jet and photo-pion decay from $\mathrm{p} \gamma$ interactions where

\footnotetext{
${ }^{1}$ Instituto de Astronomía, UNAM, 04510, México; e-mail: jsacahui@astro.unam.mx; magda@astro.unam.mx; nfraija@astro.unam.mx; joselo@ciencias.unam.mx;

wlee@astro.unam.mx
} 
protons are accelerated at the shocks and photons come from the prompt emission or an external radiation field. On leptonic models the HE emission is proposed to be IC, SSC and synchrotron emission of accelerated electrons in internal (Panaitescu \& Meszaros 2000; Papathanassiou \& Meszaros 1996) or external (Sari et al. 2001; Wang et al. 2001a) shocks.

GRB 980923 (González et al. 2012) and GRB 090926A (Ackerman et al. 2011) presented a short $\mathrm{MeV}$-peak and a keV-tail with longer duration than the prompt emission. The MeV-peak have not been reported in other bursts. GRB 090926A showed a long lasting GeV-component. We have pointed (Fraija et al. 2013) some similarities and differences between these bursts and developed a model (Fraija et al. 2012a,b; Sacahui et al. 2012) to described the HE components apparent in both bursts.

Fraija et al. (2012) and Sacahui et al. (2012) described for GRB 980923 and GRB 090926A respectively, the short MeV-peak in both bursts and the long-lasting GeV high-component in GRB 090926A as SSC emission from external shocks. Furthermore, they explored synchrotron emission in a unified way to explain the observed tail at lower energies. In both GRBs, different equipartition parameters were considered for the reverse and forward shocks $\left(\epsilon_{B, r} \neq \epsilon_{B, f}\right.$ and $\left.\epsilon_{e, r} \neq \epsilon_{e, f}\right)$, leading to a highly magnetized ejecta.

On the other hand, GRB 940217 was one of the longest and also the most energetic burst seen by the Compton Telescope (COMPTEL) (Winkler et al. 1995). It was also detected, the Energetic Gamma-Ray Experiment Telescope (EGRET) (Winkler et al. 1995) and Interplanetary Network (Ulysses/Burst and Transient Source Experiment, BATSE) (Hurley et al. 1994). The duration of the bursts given by T90 was $162 \mathrm{sec}$ as reported by Winkler et al. (1995). The total fluence above $20 \mathrm{keV}$ was $(6.6 \pm 2.7) \times 10^{-4} \mathrm{erg} \mathrm{cm}^{-2}$, as observed by BATSE large area detectors (Hurley et al. 1994). The EGRET spark-chamber recorded 10 photons while the main emission was in progress. Following this, an additional 18 photons were recorded for $\sim 5400 \mathrm{~s}$, including an $18 \mathrm{-GeV}$ photon $\sim 4500 \mathrm{~s}$ after the main emission had ended. The COMPTEL Telescope observed Winkler et al. (1995) GRB 940217 in the energy range of $0.3-30 \mathrm{MeV}$. Five episodes were identified in the reported light curve. In particular, a fifth episode or peak at UT $=83088.75 \mathrm{~s}$ (burst trigger at UT $=82962 \mathrm{~s}$ ) with a duration of $18 \mathrm{~s}$ is observed only at energies higher than $30 \mathrm{MeV}$. This short peak was described as a simple power law with a spectral index of $1.78_{-0.38}^{+0.52}$ (Winkler et al. 1995).

In this work we apply the model presented by Fraija et al. (2012) and Sacahui et al. (2012) on GRB 940217 to describe its high-energy components. We introduce standard values for the input parameters and obtain break energies, fluxes, duration, etc. in agreement with the observed values.

\section{External shock leptonic emission}

We use the model described by Fraija et al. (2012) and Sacahui et al. (2012). Here, we present a brief summary of the model. For a detailed description of the model see (Fraija et al. 2012a; Sacahui et al. 2012). 
In the external shock model, GRB emission is produced when an expanding relativistic shell interacts with the circumburst medium producing forward and reverse shocks. In addition to the dynamics of the forward shock, a reverse shock propagating back into the shell is expected, which would decelerate or not the shell depending on the evolution of the reverse shock: thick or thin shell respectively. For the forward shock, we assume that electrons are accelerated to a power-law distribution of Lorentz factors $\gamma_{e}$ with a minimum Lorentz factor $\gamma_{m}: N\left(\gamma_{e}\right) d \gamma_{e} \propto$ $\gamma_{e}^{-p} d \gamma_{e}$ where $\gamma_{e} \geq \gamma_{m}$ and $\epsilon_{e, f}$ and $\epsilon_{B, f}$ are the constant fractions of the shock energy that is transferred into the electrons and the magnetic field, respectively. These equipartition parameters are given by: $\epsilon_{e, f}=U_{e} /\left(4 \gamma_{f}^{2} \eta_{f} m_{p}\right)$ and $\epsilon_{B, f}=$ $B_{f}^{2} /\left(32 \pi \gamma_{f}^{2} \eta_{f} m_{p}\right)$ where $\gamma_{f}$ is the bulk Lorentz factor and $\eta_{f}$ is the density of the surrounding medium.

Given the cooling electron Lorentz factor $\gamma_{e, c}=3 m_{e}(1+z) /\left(16 \epsilon_{B, f} \sigma_{T} m_{p} t_{d, f} \Gamma_{f}^{3} \eta_{f}\right)$ and the deceleration time $t_{d, f}=(1+z)\left(3 E / 32 \pi m_{p} \eta_{f} \Gamma_{f}^{8}\right)^{1 / 3}$, the highest break energy and maximum flux of the synchrotron photons radiated by electrons at a distance $D$ from the source in natural units are given by,

$$
\begin{aligned}
E_{\mathrm{m}, \mathrm{f}} & \sim \frac{2^{5 / 2} \pi^{1 / 2} q_{e} m_{p}^{5 / 2}(p-2)^{2}}{m_{e}^{3}(p-1)^{2}}(1+z)^{-1} \epsilon_{e, f}^{2} \epsilon_{B, f}^{1 / 2} n_{f}^{1 / 2} \gamma_{f}^{4} \\
F_{\max , \mathrm{f}} & \sim \frac{m_{e} \sigma_{T}}{36 \pi m_{p}^{1 / 2} q_{e}}(1+z) \epsilon_{B, f}^{1 / 2} n_{f}^{1 / 2} D^{-2} E
\end{aligned}
$$

where $E$ is the isotropic energy. Now, electrons in the forward shock region can upscatter the synchrotron photons in accordance to the following equations: $E_{m, f}^{(\mathrm{SSC})} \sim \gamma_{m}^{2}, E_{m, f}$. Then the SSC break energy is given by,

$$
E_{\mathrm{m}, \mathrm{f}}^{(S S C)} \sim \frac{6 q_{e} m_{p}^{15 / 4}}{2^{5 / 4}(3 \pi)^{1 / 4} m_{e}^{5}}(1+z)^{5 / 4} \epsilon_{e, f}^{4} \epsilon_{B, f}^{1 / 2} n_{f}^{-1 / 4} E^{3 / 4} t_{f}^{-9 / 4} .
$$

When the reverse shock crosses the shell it heats up and accelerates electrons. In the thick shell case the reverse shock becomes relativistic during the propagation and the shell is significantly decelerated. The crossing time of the reverse shock might be much smaller as long as the $\sigma$ magnetization parameter increases, thus for $\sigma \sim 1$, the time for the short MeV-peak would be $t_{d} \sim T_{90} / 6$, which could be in agreement for bright GRBs according to Lamb et al. (2005).

For the reverse shock the maximum flux, break energy of synchrotron and SSC break energy are given by,

$$
\begin{aligned}
E_{\mathrm{m}, \mathrm{r}} & \sim \frac{4 \pi^{1 / 2} q_{e} m_{p}^{5 / 2}(p-2)^{2}}{m_{e}^{3}(p-1)^{2}}(1+z)^{-1} \epsilon_{e, r}^{2} \epsilon_{B, r}^{1 / 2} \Gamma_{r}^{2} n_{r}^{1 / 2} \\
F_{\max , \mathrm{r}} & \sim \frac{m_{e} \sigma_{T}}{2^{3 / 4} 36 \pi m_{p}^{1 / 2} q_{e}}(1+z)^{7 / 4} \epsilon_{B, r}^{1 / 2} n_{r}^{1 / 4} D^{-2} E^{5 / 4} \Gamma_{r}^{-1} T_{90}^{-3 / 4} \\
E_{\mathrm{m}, \mathrm{r}}^{(S S C)} & \sim \frac{2^{21 / 4} \pi^{3 / 4} m_{p}^{13 / 4}(p-2)^{4}}{3^{1 / 4} m_{e}^{5}(p-1)^{4}}(1+z)^{-7 / 4} \epsilon_{e, r}^{4} \epsilon_{B, r}^{1 / 2} \Gamma_{r}^{4} n_{r}^{3 / 4} E^{-1 / 4} T_{90}^{3 / 4}
\end{aligned}
$$


we have used the equations given above to calculate the duration of the HE components, their break energies and the energy fluxes associated to the break energies. The parameters used for forward and reversed shocks are given in Table 1. A search in the parameter space to look for possible solutions was performed. The calculated values are given in Table 2, for comparison the observed maximum energies, the associated energy fluxes and the durations of the components are also given. We also show the solutions for GRB 090926A and GRB 980923 as reported by Fraija et al. (2012) and Sacahui et al. (2012) for comparison.

Table 1. Parameters used.

\begin{tabular}{lccc}
\hline GRBs & 940217 & 980923 & $090926 \mathrm{~A}$ \\
\hline Forward shock & & & \\
$\epsilon_{B, f}$ & $10^{-4}$ & $10^{-5}$ & $10^{-4.3}$ \\
$\epsilon_{e, f}$ & 0.35 & 0.95 & 0.1 \\
$n_{f}\left(\mathrm{~cm}^{-3}\right)$ & 10 & 1 & 10 \\
$\gamma_{f}$ & 600 & 600 & 600 \\
\hline Reverse shock & & & \\
$\epsilon_{B, r}$ & 0.125 & 0.125 & 0.125 \\
$\epsilon_{e, r}$ & 0.35 & 0.6 & 0.65 \\
$n_{r}\left(\mathrm{~cm}^{-3}\right)$ & 10 & 10 & 10 \\
$\gamma_{r}$ & 1000 & 1000 & 1000 \\
\hline
\end{tabular}

Table 2. Calculated quantities using the model described in the text. When available, the observed values are given.

\begin{tabular}{|c|c|c|c|}
\hline GRBs & 940217 & 980923 & 090926A \\
\hline Quantities & $\begin{array}{l}\text { calculated } \\
\text { (observed) }\end{array}$ & $\begin{array}{l}\text { calculated } \\
\text { (observed) }\end{array}$ & $\begin{array}{l}\text { calculated } \\
\text { (observed) }\end{array}$ \\
\hline \multicolumn{4}{|l|}{ Forward shock } \\
\hline$E_{\mathrm{m}, \mathrm{f}}(\mathrm{keV})$ & $175.5(-)$ & $78.9(128.5)$ & $10.13(\sim 50)$ \\
\hline$E_{\mathrm{m}, \mathrm{f}}^{(S S C)}(\mathrm{GeV})$ & $10.9(\sim 18)$ & $-(-)$ & $18.4(\sim 10)$ \\
\hline $\begin{array}{l}\text { Duration of } \\
\text { the component (s) }\end{array}$ & $1000(\sim 5600)$ & $-(-)$ & $100(\sim 100)$ \\
\hline $\begin{array}{l}\left(\nu F_{\nu \max }\right)^{S S C} \\
\left(\operatorname{erg~cm}^{-2} \mathrm{~s}^{-1}\right)\end{array}$ & $2.52 \times 10^{-6}\left(\sim 10^{-6}\right)$ & $1.2 \times 10^{-7}\left(\sim 10^{-6}\right)$ & $1.09 \times 10^{-6}\left(\sim 10^{-6}\right)$ \\
\hline \multicolumn{4}{|l|}{ Reverse shock } \\
\hline$E_{\mathrm{m}, \mathrm{r}}(\mathrm{eV})$ & $47.9(-)$ & $0.14(-)$ & $0.17(-)$ \\
\hline$E_{\mathrm{m}, \mathrm{r}}^{(I C)}(\mathrm{MeV})$ & $34.8(\geq 30)$ & $427.2(\geq 150)$ & $414.3(400)$ \\
\hline $\begin{array}{l}\text { Duration of } \\
\text { the component (s) }\end{array}$ & $27(\sim 7)$ & $6(\sim 2)$ & $6(\sim 1)$ \\
\hline $\begin{array}{l}\left(\nu F_{\nu \max }\right)^{S S C} \\
\left(\operatorname{erg~cm}^{-2} \mathrm{~s}^{-1}\right)\end{array}$ & $3.34 \times 10^{-7}\left(\leq \times 10^{-6}\right)$ & $2.2 \times 10^{-6}\left(\sim 10^{-6}\right)$ & $8.2 \times 10^{-6}\left(\sim 10^{-6}\right)$ \\
\hline
\end{tabular}




\section{Results and discussion}

The parameters used to describe these components are similar to the ones found for both GRB 980923 (Fraija et al. 2012a,b) and GRB 090926A (Fraija et al. 2012c; Sacahui et al. 2012), as expected because of the similarities in the observables (spectral index, energy ranges, etc.). Our model is very dependent on the value of $\epsilon_{B, r}=0.125$ implying a value of the magnetization parameter of $\sigma \sim 1$ and therefore a presence of a highly magnetized jet. This high degree of magnetization gives rise to a shorter duration (as compared with the burst duration) of the emission at the reverse shock in the thick shell case. The parameters used (see Table 1) to describe the observations are typical for GRBs. The current model accounts for many characteristics of the bursts: energies, spectral indices, fluxes, durations of the components in a unified manner.

In summary, we have presented a leptonic model based on external shocks to describe the short $\mathrm{MeV}$ - peak and longer lasting $\mathrm{GeV}$ emission in a unified manner for GRB 940217 as done before for GRB 980923 and GRB 090926A. These bursts seem to form a subset of bursts with a same dynamic at the jet and a magnetized ejecta.

This work is partially supported by DGAPA-UNAM (Mexico) Project No. IN105211, IN110212 and Conacyt Project No. 103520.

\section{References}

Ackerman, M., Ajello, M., Asano, K., et al., 2009, ApJ, 729, 114

Asano, K., Guiriec, S., \& Meszaros P., 2009, ApJ, 705, L191

Dermer, C.D., \& Razzaque, S., 2010, ApJ, 724, 1366

Fraija, N., Gonzalez, M.M., \& Lee, W.H., 2012, ApJ, 751, 33

Fraija, N., Gonzalez, M.M., \& Lee, W.H., 2012 [arXiv:1110.6421]

Fraija, N., Gonzalez, M.M., Sacahui, J.R., \& W.H. Lee, 2012, PoS GRB 2012, 27

Fraija, N., Gonzalez, M.M., Sacahui, J.R., et al., 2013, in preparation

González, M.M., Sacahui, J.R., Ramirez, J.R., et al., 2012, ApJ, 755, 140

Hurley, K., Dingus, B.L., Mukherjee, R., et al., 1994, Nature, 372, 652

Lamb, D.Q., Donaghy, T.Q., \& Graziani, C., 2005, ApJ, 620, 355

Panaitescu, A., \& Meszaros, P., 2000, ApJ, 544, L17

Papathanassiou, H., \& Meszaros, P., 1996, ApJ, 471, L91

Sacahui, J.R., Fraija, N., Gonzalez, M.M., et al., 2012, ApJ, 755, 127

Sari, R., Narayan, R., \& Piran, T., 1996, ApJ, 473, 204

Sari, R., \& Esin, A.A., 2001, ApJ, 548, 787

Wang, X.Y., Dai, Z.G., \& Lu, T., 2001, ApJ, 546, L33

Winkler, C., Bennett, K., Hanlon, L.O., et al., 1994, Ap\&SS, 231, 153 
